\title{
Optimizing Delivery Mechanisms in Wireless Mesh Network with QOS Constraints
}

\author{
Soma Pandey \\ Assistant Professor \\ CMR Institute of Technology \\ \#132, AECS Layout, ITPL Road, \\ Bangalore - 560037
}

\author{
Vijay Pande \\ Professor \\ CMR Institute of Technology \\ \#132, AECS Layout, ITPL Road, \\ Bangalore - 560037
}

\author{
Govind Kadambi \\ Professor and Dean \\ MS Ramaiah School of Advanced \\ Studies, New BEL Road \\ Bangalore - 560054
}

\begin{abstract}
In this paper we have considered a Wireless Mesh Network (WMN) with multiple internet gateways (IGW) and wired network support for maximizing the delivery in the WMN access network. We have considered the WMN in IEEE 802.11s framework that nodes can transit from one IGW region to the other seamlessly based on the Quality of Service (QOS) requirements. Then we set up the system and method for maximizing delivery in the last mile through wired gateways. Then subsequently we address the problem of IGW placement in the WMN.
\end{abstract}

\section{Categories and Subject Descriptors}

C.2.1 [Wireless Communication]: Design of wireless LAN, 802.11s, Combining wired and wireless networks, 802.11s networks, Wireless LAN

\section{General Terms \\ Design}

\section{Keywords}

WMN, IEEE802.11s, Planar graph, Internet Gateway Placement, Mesh Portal Point, Mesh Router, multihop wireless and wired network.

\section{INTRODUCTION}

Despite considerable research on 802.11 multihop wireless mesh networks (WMN), the scope for efficient utilization of electro magnetic spectrum remains. Because of this reason WMN is not able to match the wired network in terms of bandwidth. High speed broadband connection with seamless handoff and handover is a limitation for $3 \mathrm{G}$ and $4 \mathrm{G}$ wireless systems. In this paper we have tackled the problem of channel assignment in an entirely different way. We have reduced the need of radio spectrum by providing increased wired backhauls. This has not only simplified the channel assignment problem but also has paved way for a simplified representation of the mesh. Mesh simplification leads to faster routing calculations and simpler and smaller spanning trees. Increasing wired backhauls leads to greater number of internet gateways which we call as virtual gateways. Here we presume that every wired backhaul leads to Internet gateway. In this work we have laid emphasis on the main drawback of wireless LANs whereby their bandwidth decreases by $1 / 2^{n}$ with increase in no. of hops from the gateway, where $n$ is the no. of hops [5]. We introduce more number of gateways in the network such that they not only simplify the mesh topology but also dynamically share load among themselves without the core network intervention. In first section we explain the related work done in this area. Next we present our network model based on planar graph, thereafter we derive some results which we use in our next paper to give an algorithm which simplifies the mesh, reduces routing computation and Minimum Spanning Tree calculations. Thereafter in coming papers we devise our load distribution algorithm which enables the nodes to dynamically detach and attach themselves from gateways without disturbing the hierarchy of layers.

\section{RELATED WORK}

In the past lot of work has been done on WMN and Internet gateway (IGW) deployment problem. IGW deployment problem are addressed in [1] by Xie et al where in deployment problem is addressed as Linear Programming (LP) problem, this is extendable even to mesh network formed on $802.11 \mathrm{a} / \mathrm{b} / \mathrm{g}$ frame work. Prior to this work Bejerano et al [2] also address the problem of (IGW) placement, where in they consider WMN as a large scale network mapped into graph(clusters) and then divided graphs into disjoint sub graph (disjoint cluster) rooted at each IGW for the large scale last mile network. In their work a good framework is laid for the integration of Multihop wireless and wired networking the last mile. But Bejerano work is mainly focused on wireless sensor networks. Their network model does not consider any of IEEE 802.11 standards as basis of. If we take a look into network model, an IGW is considered to be connected through wired network to the internet space, this means that only limited QOS is achieved secondly it does not have any mechanism to dynamically carry out load balancing among the IGWs. IEEE 802.11s standard allows the Mesh Router (MR) to transit based on the traffic or QOS condition to other IGW region. In light of this we formulate the problem for increased delivery mechanism in the last mile and setting up auxiliary problem as IGW placement and converting ordinary 
MR to virtual IGW in case of wired MR. In this paper we are concerned about maximizing the delivery in the WMN not by just placing IGW but by positioning wired network in such a way that MR can be scaled to IGW. We have considered IEEE 802.11s framework as it paves the way for MR to transit from one IGW to the other IGW region seamlessly. We used this fundamental as basis for IGW placement. From the practical stand point, the fundamental requirement is the delivery/throughput with QOS in the mesh, as increase or decrease in the number of IGW may not guarantee the throughput at all MRs/MPP. So it is important to address the problem of WMN from the point of enhancing the delivery system with QOS as constraints.

\section{PREAMBLE}

We address the problem in following way. We assume that, an IEEE802.11s WMN framework consists of node $\mathrm{V}_{\mathrm{i}}$ that can be MAP/MP/MR/MPP/IGW [3]. Some of the nodes are made as MPP/IGW connected to the core network through wired or wireless network. Excessive Placement of IGW is done with a view that QOS/bandwidth constraints need to be met. Mesh formation at each MR/MAP/MPP happens on a dedicated radio with a consideration that the channels are available. The practical considerations can be considered in the simulation. Then around each IGW a polytopic region comprising of set of neighboring $\mathrm{V}_{\mathrm{i}}$, is created such that QOS criteria is satisfied. Considering wired medium of the backhaul for MR makes MR as IGW in WAN/LAN format implies that arbitrary large number of IGW can be formed from MR/MAP. This means managing IGW with nodes for QOS needs formulation. Load balancing in form of dropping nodes and adding nodes to wherein it can be catered. Interestingly this is defined in 802.11 s. In this paper we create this framework and prove that a great simplification can be achieved. Any generic WMN can be represented by directed graph with edges as communication links, and vertices as nodes representing MPP (IGW)/MAP/MP (MR). For incoming traffic gateway becomes source node and MAP/MP becomes sink node. For outgoing traffic MAP/MP becomes source and MPP becomes sink. Outgoing traffic in $W M N$ has many sources and one sink while incoming traffic to WMN has one source and many sink. We in paper refer nodes as MR or MP. In graph representation MP, MAP, MPP and nodes are also referred as vertices and feasible link among the vertices are referred as edge.

\section{MODEL}

Each node can be represented by graph. The hopping sequence under various constraints is a cyclic walk. i.e. alternate between edges and vertices. Larger the acyclic walk higher is the bandwidth loss. Lot of literature is available that work out time complexity of graph or polytope. In our work our prime focus is to work upon enhancement of bandwidth by following propositions.

1. Adding sources in the mesh.

2. Adding different type of sources and

3. Adding more nodes so as to handle traffic at the cost of computation complexities. In graph literature also called as Steiner nodes
4. Simplification by virtue of graph contraction method as an outcome of adding different type of sources (as mentioned in proposition-2) in to reduce the number of hops in mesh.

5. Generate metric for defining the capacity of mesh under one source with maximizing bandwidth by construction of polytope with one node as source. This we call as span of gateway. What size of polytope should be catered by each source? Defining span of gateway in polytopic sense provides a methodology of adding or deleting sinks from mesh thereby not changing any structure

6. Span of gateway in polytopic way can be defined as intersection of polytopes where in common node among them remains for dynamic position in the polytope. Hence only common nodes need to be positioned by looking in to the capacities of gateways. The objective here is to reduce the hops, generate more capacity in the network (hop reduces bandwidth of the network and additional source gateway increases the bandwidth of network with increase in computational complexities)

7. Placement of powerline gateway in the mesh will bring in further simplification into the polytopic structure under each gateway. (This is outcome of proposition 3 and 5).

In this paper we consider model derived from Xie et al and is expressed below. In this model we follow IEEE-802.11s, and subsequently we further enhance the model to incorporate MR on wired network with connectivity to WMN. Here MR shall be on LAN/WAN with wireless on IEEE802.11a/b/g/s interconnect in the mesh. There are two significant simplifications that can be brought forward in the mesh network.

1. It paves way for augmenting wired medium into mesh network arbitrarily thereby augmenting MR conversion to IGW in the mesh and improving system delivery mechanism at the increased cost of computation at the server side.

2. It brings simplification into WMN graph by virtue of contraction and reducing the edges and vertices in the wmn planar graph. Planarity ensures polytopic structure.

Usually in the literature tree formed from the mesh is rooted graph with IGW as root and IGW placement problem is described on the tree structure. Then one has to work on finding minimal number IGW placement at the nodes on the tree that will meet QOS. Then disjoint partitions are created on the rooted tree and within boundary of partition, QOS is met at all the nodes. In such approach, the excess capability of some of the partitions and failing nodes in the other partitions or increasing load in other partition may not satisfy the QOS. We introduce concept of wired medium access in WAN/LAN on one side and WMN on other side. This augmentation of wired medium set the delivery mechanism in mesh network supplemented by a graph.

\section{NOTATIONS AND REPRESENTATIONS}


Let $V i$ represents the node of the $W M N$ where in each node can be $M P P / M A P / M P$. Consider that $W M N$ has multiple gateways (ie multiple $M P P$ ) in the mesh where in few of the nodes acts like source/MPP (this is a generic consideration in a 802.11 s).

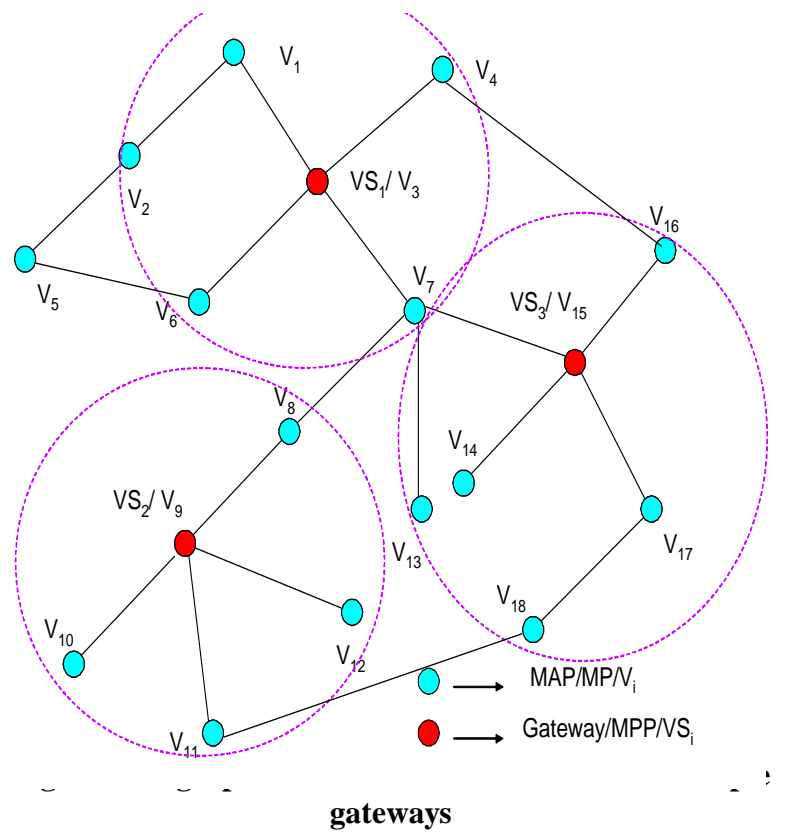

Consider there are $\underline{\boldsymbol{n}}$ vertices and $\underline{\boldsymbol{m}}$ edges/links connecting them. Hence WMN can be represented as

$\mathrm{WMN}$ is $\bigcup_{i, j}^{n, m}(V i, E j)$, where its adjacency matrix can be denoted as $[\boldsymbol{A W}]_{m \text { is } n \times \mathrm{x} n \text { matrix and the incidence }}$ matrix is denoted by $[W]_{\text {is } m \times n \text { matrix. Consider that }}$ there are $\boldsymbol{q}$ source in the mesh network. Example in figure $1 \mathrm{q}=$ 3 Here $n, m, q \in Z$. $\exists n>q$ •

For the sake of simplicity all source set can be specially denoted as $\boldsymbol{V S i}$

$$
V S_{i} . \forall i \in \mathcal{1}_{3} . . q_{\text {and }}\{V S\} \subset\{V\} \text {. }
$$

Now consider $P_{i}$ as polytope with one $V S_{i}$ as vertex, of dimension $\boldsymbol{k}$, hence there can be at most $\boldsymbol{q}$ polytopes. Also it means each polytope $P_{i}$ has one source.

It means one of the nodes $V_{k}$ in $P_{i}$ as gateway (source) represented as $V S i$. Then all feasible spanning tree enumeration $S_{e}$ with $V S_{i}$ as source (gateway) in a polytope $P_{i}$ be $\bigcup_{k=1}^{p} S_{k}$

where $p$ are number of nodes/vertex in the polytope and $S$ as spanning tree. Here

$\min \left|\mathbf{S}_{e}\right| \forall k=(1 \ldots p)$ is a minimum spanning tree for

$P_{i}$ $\max \left\lfloor S_{e}^{i} \mid \forall k=1 \ldots p\right.$ and $\max \left[S_{e}\right] \forall \mathrm{k}=1 . . \mathrm{p}$ with weights on edges and condition on (p) vertices for $P_{i}$.

Then the set of all spanning tree for entire (mesh) polytope $\bigcup_{i=1}^{q} S_{e}^{i} . . \forall k=1 \ldots . . p$

Consider a mesh network with multiple mesh gateway connected to some backend core network, then the above propositions can be set into the following objective

1. Find the subnet of mesh that can be fed by gateway satisfying the constraints of QoS and mobility (sizing problem)

2. Find out the mathematical basis to improve the QoS relatively by positioning heterogeneous gateway into the same mesh (source positioning problem)

3. Study the performance of such systems from QoS and mobility standpoint simulation - showing the performance. (simplification quantification problems)

\section{MATHEMATICAL RESULTS}

Lemma 1 : Mesh network is a polytope of dimension equal to no of available channel.

Proof: Entire mesh can be represented as vertices and each channel connecting one vertex to another vertex as links are edges. Now consider that there are three nodes with one source then linear can represent a polytope. Now we need to prove the sufficiency. Now consider a node inside a polytope such node can be represented by linear combination of other node (vertices). Now consider the node outside the polytope, then with the links from the adjacent nodes connect a new polytope is formed, hence mesh is always a polytope

Theorem 1 : Consider a mesh with $\underline{\boldsymbol{n}}$ vertices and $\underline{\boldsymbol{p}}$ edges, consider $\boldsymbol{q}$ source nodes of same type in a same mesh then $\boldsymbol{q}$ 
source can have $\boldsymbol{q}$ polytopes, then if $\boldsymbol{q}$ polytopes have QoS satisfied then entire mesh satisfies QoS limits.

Proof: As per lemma-1, if $\max \left[S_{e}\right] \forall \mathrm{k}=1$..p QOS is satisfied then that polytope $Q o S$ is met and if same is achieved for $\max \left\lfloor\mathbf{S}_{e}^{i}\right\rfloor \forall k=1 \ldots p$

hence QOS is achieved for entire mesh.

Implication of this theorem is that it is a stiff sufficient condition for achieving $Q o S$ for the mesh network, but can be good starting point for reducing the stiffness of sufficient condition.

Theorem-2: In continuation with above theorems $\bigcup_{i=1}^{q} P i$ is an original mesh. A non empty intersection of any $\bigcap_{i=1}^{q} P_{i}, \forall i \in(1 \ldots q)$

dimension.

polytope is a polytope at most of same

Proof: mesh is collection of defined polytopes. Since $\boldsymbol{q}$ polytopes are formed from the mesh then collection of $\left[P_{i} \forall \mathrm{i}=1 . . \mathrm{q}\right]$ polytopes is mesh. Since any two or more polytope in $P_{i}$ set can have common nodes. These common nodes in structure can also be represented as polytope. Consider

$\left\{Q_{j}\right\}=\left\{\bigcap_{i=1}^{q} P_{i}, \forall i \in(1 \ldots q)\right\}$ be non empty intersection of polytopes. Then $Q_{j}$ are also a polytopes from 1 to full dimension. These polytopes contains vertices which can transit into the other polytopes based on the condition defined.

Corollary:1 (of theorem-1) If $\bigcup_{i=1}^{q} P i$ is a complete mesh and if $\min \left|\mathbf{S}_{e}^{i}\right| \forall k=1 \ldots p$

achieved for entire mesh if and only if $\bigcap_{i=1}^{q} P_{i}=\phi, \forall i \in(1 \ldots q)$

Proof: All possible intersection of polytope is equivalent to all sub graphs as an independent graph. Since necessary condition for QoS is that minimum spanning tree is having QoS and all sub graphs have QoS. For sufficiency, no two graphs have any vertices or node in common.

Corollary-2 (derived from Kurtowaski and Wagner theorem): Consider a mesh $\bigcup_{i=1}^{q} P_{i}$ then contraction of two adjacent nodes reduces the cycle by 1 and reduces the edges by 2 , and maintains the planarity of the graph.

Proof: Consider $\boldsymbol{V}_{\boldsymbol{i}}, \boldsymbol{V}_{\boldsymbol{j}}$ as adjacent node in a cycle denoted as $Z_{i} \in P_{i}$ then if $V_{\boldsymbol{x}} \approx\left(\boldsymbol{V}_{\boldsymbol{i}} \equiv \boldsymbol{V}_{\boldsymbol{j}}\right)$ reduces 2 edges and can reduce cycle. If a cycle consists of three vertices then cycle is reduced if cycle is of 4 vertices then contraction of two vertices reduces cycle length to three and for $n$ vertices in a cycle its length reduces by $n-1$.

Theorem-3: Consider a mesh with $\underline{\boldsymbol{n}}$ vertices and $\underline{\boldsymbol{p}}$ edges, also consider $\boldsymbol{q}$ source node of same type in mesh, consider that the $\boldsymbol{V}_{\boldsymbol{i}}$ node and $\boldsymbol{V}_{\boldsymbol{j}}$ node are contracted in the mesh then there exist much simpler sufficient condition such that QoS limits are satisfied for entire mesh.

Proof: Consider that $\boldsymbol{V}_{\boldsymbol{i}}, \boldsymbol{V}_{\boldsymbol{j}} \in \boldsymbol{P}_{a} \Rightarrow \boldsymbol{V}_{\boldsymbol{i}}, \boldsymbol{V}_{\boldsymbol{j}}$ are nodes of same sub graph, then contraction of $\boldsymbol{V}_{\boldsymbol{i}}, \boldsymbol{V}_{\boldsymbol{j}}$ ( are adjacent nodes) results in reduction in walk there by reducing the $\boldsymbol{S}_{\boldsymbol{e}}$ length. Now consider that $\boldsymbol{V}_{\boldsymbol{i}} \in \boldsymbol{P}_{\boldsymbol{a}}$ and $\boldsymbol{V}_{\boldsymbol{j}} \in \boldsymbol{P}_{\boldsymbol{b}}$ then after contraction

$\boldsymbol{V}_{\boldsymbol{x}} \approx\left(\boldsymbol{V}_{\boldsymbol{i}} \equiv \boldsymbol{V}_{\boldsymbol{j}}\right)$ become a common node to both $\boldsymbol{P}_{a}$ and $\boldsymbol{P}_{\boldsymbol{b}}$. If $V_{x}$ is dropped from say $\boldsymbol{P}_{\boldsymbol{a}}$ then again walk is reduced for $\boldsymbol{P}_{\boldsymbol{a}}$ and walk is also reduced in $\boldsymbol{P}_{\boldsymbol{b}}$. (Corollary - 2) This is simpler sufficient condition as walks are reduced.

Proposition 1: $\quad T w o$ adjacent nodes $\boldsymbol{V}_{\boldsymbol{i}}, \boldsymbol{V}_{\boldsymbol{j}}$ can be contracted without loss in generality of mesh. This contraction can be used in source placement problems in the mesh.

Proposition 2: Any node $\boldsymbol{V}_{i}$ can become a source node VSi without loss in generality of mesh

Extension of the work as source placement problem in a mesh can solve bandwidth related issues.

Theorem-4: The transiting vertices of the $q$ polytopes for managing QoS limits are the non empty intersection of any $\boldsymbol{q}$ polytopes only. If QoS limits are satisfied for the transiting polytope then QoS is achieved for entire mesh.

Proof: Polytopes are planar graphs implies no two edges can intersect, this means that no cross over of communication link can happen in the same mesh, which is generic and practical condition. Planar graph, which we are referring as polytope are the graphs in which no two cycles are intersecting. Let

$$
\text { Q } 1 \bigcap_{j=1}^{q} P_{i} \neq \phi, \forall i \in(1 \ldots q) \text { be the nodes of all }
$$
transiting polytopes. If QoS is satisfied for $\boldsymbol{Q}_{j}$ then entire $\bigcup_{i=1}^{q} P i$ have QoS satisfied.

Corollary-3: Consider that out of $\boldsymbol{q}$ source, only $\mathbf{q - 1}$ sources are active then $\boldsymbol{q}^{\text {th }}$ polytope is an intersection of $\boldsymbol{q}-1$ polytopes. (This is a corollary to theorem 3 and 4). 
Proof: If $\boldsymbol{q}^{\text {th }}$ source is just another node then $\boldsymbol{P}_{\boldsymbol{q}} \subset \bigcup_{i=1}^{q-1} P i$ ( $\forall i=1 \ldots q-1)$. If it is not a subset then case becomes trivial.

A WMN is represented as<smiles>[Te]C1CC2CCC2C1</smiles>

Then adjacency matrix $[\mathrm{A}(\mathrm{G})]$ is $\mathrm{n} \times \mathrm{n}$ matrix represented by mesh access points or gateways. Let [IW (G)] be incidence matrix of $\mathrm{n} \times \mathrm{m}$ matrix (representing connections). Consider that there are $\boldsymbol{k}$ gateways in the mesh represented by some of $V_{i} \forall i \in$ $[1 \ldots k]$ then exist transformation. Let $[\mathrm{C}(\mathrm{G})]$ be a cycle matrix of the mesh of $1 \times \mathrm{m}$

Theorem 5: For a simple mesh (WMN) represented by a planar graph $G$. Consider a function $f$ such that

$f:[I W(G)] \rightarrow\left[\begin{array}{ccc}{\left[I W\left(G_{1}\right)\right]} & 0 \\ & \ddots & \\ 0 & & {\left[I W\left(G_{k}\right)\right]}\end{array}\right]$

Then if $[I W(G)][C(G)]^{T}=0(\bmod 2)$ then their exist

$C(G) \rightarrow\left[\begin{array}{ccc}C\left(G_{1}\right) & & 0 \\ & \ddots & \\ 0 & & C\left(G_{q}\right)\end{array}\right]$

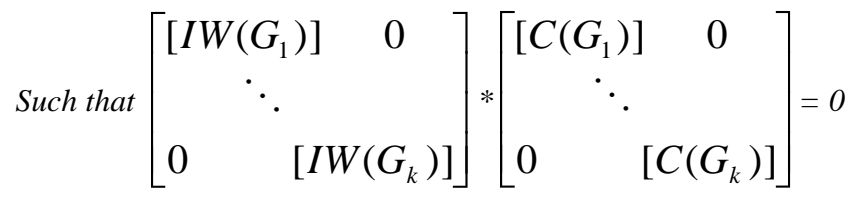

And

$\left[I W\left(G_{i}\right)\right] *\left[C\left(G_{i}\right]^{T}=[0]\right.$

And rank of $\left[\operatorname{IW}\left(G_{i}\right)\right]$ is $\boldsymbol{n}-\boldsymbol{k}$ and $\left[C\left(G_{i}\right]\right.$ is $\boldsymbol{l}-\boldsymbol{n}+\boldsymbol{k}, \boldsymbol{l}$ is rank of $[C(G)]$

Proof: Shall be presented in next paper
As a consequence of above theorem, mesh will have following impact on its adjacency matrix, where in each $\left[A\left(G_{i}\right)\right]$

contains one IGW. This means that each $\left[A\left(G_{i}\right)\right]$

is a disjoint set containing one IGW, and function $f$ implies removal or addition of edges (connecting channels) with balancing cycle matrix as of theorem-5. Each $\left[A\left(G_{i}\right)\right]$ with one IGW satisfies QOS for

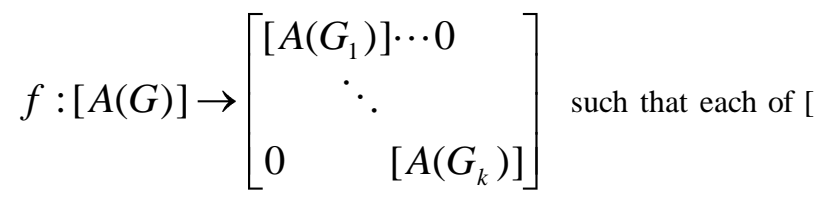

A $\left(\mathrm{G}_{\mathrm{i}}\right)$ ] contains one gateway each where the rank $[A(G)] \geq \sum_{i=1}^{k} \operatorname{rank} A\left[G_{i}\right]$

\section{DELIVERY MAXIMZATION WITH IGW (WIRED) PLACEMENT ALGORITHM}

Based on the above mentioned theorem, an algorithm is formulated, which shall be presented as separate paper.

\section{SIMULATION RESULTS}

For a very large scale WMN i.e. very large number of MR, IGW and capacity, following are simulation results.

1. Capacity of each IGW with number of wired MR.

2. Number of IGW (wireless) by varying MR-IGW (wire line) through put.

3. Throughput capacity of IGW on number of node drop MR-IGW (wire line).

4. Number of IGW nodes to MR through put capacity.

5. And then comparison on different IGW throughput capacity (wired and wireless).

(Shall be presented as separate paper)

\section{CONCLUSION}

In this paper we have build an innovative method to improve capacity of WMN. Enhancing methods are simple and geometric in nature. We formulated a delivery enhancement and IGW placement problem as multi Linear Programming problem. The solution of such problem is by means of polytopic constructions of nodes.

\section{ACKNOWLEDGMENTS}

Our sincere thanks to Dr. KVS Anand Babu, Principal, CMRIT for his continuous guidance and support.

\section{REFERENCES}

[1] Bing He, Bin Xie, and Dharma P. Agrawal, "Optimizing the Internet Gateway Deployment in a Wireless Mesh Network", Proceedings of IEEE MASS 2007, October 2007.

[2] Yigal Bejerano"Efficient integration of multi-hop wireless and wired networks with QoS constraints",September 2328, 2002, Atlanta, Georgia, USA 
[3] Akyildiz, I.F. and Wang, X., “A survey on wireless mesh networks”. IEEE Comput. Mag. v43 i9. 23-30.

[4] A. Raniwala, T. Chiueh, "Architecture and algorithms for an IEEE 802.11-based multi-channel wireless mesh network", in: Proceedings of IEEE INFOCOM 2005, vol. 3, pp. 22232234.

[5] Gupta, P. and Kumar, P.R., "The capacity of wireless networks". IEEE Trans. Inform. Theory. v46 i2. 388-404.

[6] H.Hrasnica, R. Lehnert, Powerline Communications in Telecommunication Access Area (Powerline Communications im TK-Zugangsbereich), VDE World Microtechnologies Congress - MICRO.tec 2000 - ETGFactagungund- Forum: Verteilungsnetze im liberalisierten Markt - Spectember 25-27, 2000 - Expo 2000, Hannover, Germany. 